REGARDS

SUR L'ECONOMIE ALLEMAND

BULLETIN ECONOMIQUE DU CIRAC
Regards sur l'économie allemande

Bulletin économique du CIRAC

$66 \mid 2004$

Varia

\title{
Le défi européen
}

Isabelle Bourgeois

\section{CpenEdition}

Journals

Édition électronique

URL : http://journals.openedition.org/rea/3845

DOI : $10.4000 /$ rea. 3845

ISBN : 978-2-8218-0828-7

ISSN : 1965-0787

Éditeur

CIRAC

Édition imprimée

Date de publication : 1 mai 2004

Pagination : 3-4

ISSN : 1156-8992

Référence électronique

Isabelle Bourgeois, "Le défi européen », Regards sur l'économie allemande [En ligne], 66 | mai 2004, mis en ligne le 12 octobre 2009, consulté le 15 septembre 2020. URL : http://journals.openedition.org/rea/ 3845 


\section{Le défi européen}

La timide reprise qui s'est esquissée à la fin 2003 semble se confirmer au début 2004, malgré quelques ombres au tableau. L'optimisme des milieux d'affaires (indice ifo), qui ne cessait de croître pendant neuf mois consécutifs, a légèrement fléchi en février, puis en mars. Au prix du pétrole et à la parité $€ / \$$ s'ajoutent des facteurs psychologiques dont une certaine impatience sur le rythme de la reprise. SeIon les différents instituts de conjoncture, l'attentat de Madrid a joué un rôle très secondaire (ifo) en comparaison de négociations salariales « dommageables à l'emploi » (ZEW) et d'une opposition croissante aux réformes engagées par le gouvernement Schröder. C'est assurément ce dernier point, lié à l'incertitude sur la poursuite de l'Agenda 2010, qui a pesé sur le moral des patrons (le chancelier n'a présenté son programme que le 25 mars), de même que la faiblesse persistante de la demande intérieure. En ce sens, l'indice ifo reflète le climat intérieur d'incertitude bien plus que l'évolution réelle des affaires.

Car selon les indicateurs disponibles avant la publication du Rapport de printemps des six Instituts de conjoncture, repoussée au 27 avril, le moteur des exportations monte en régime, avec $+5,4 \%$ entre février 2003 et 2004 , les importations ne progressant que de 3,5\% en valeur dans le même temps (Destatis). II tire les entrées de commandes dans l'industrie et la production industrielle. Quant aux investissements en biens d'équipement, ils redémarrent : au dernier trimestre 2003, et pour la première fois depuis 2001, ils avaient augmenté (+1,7\%). En janvier, la production de biens d'investissement a augmenté de 1,5\%. Les prévisions d'automne des Instituts pour 2004 (+1,7\% pour l'investissement sur l'ensemble de l'année) pourraient dès lors se confirmer.

Reste la mollesse de la demande intérieure qui freine la production de biens de consommation et, plus généralement, ralentit la reprise des activités outre-Rhin. Le haut niveau de chômage (4,56 millions de chômeurs en mars) freine toujours la consommation des ménages, dont le revenu disponible n'a guère varié, le coup de pouce fiscal étant absorbé par la hausse du budget santé. Les réformes Hartz adoptées l'an dernier ont certes accéléré la rotation entre chômage et emploi, mais sont sans effet sur la politique d'embauche des entreprises qui stabilisent, voire compriment leurs effectifs pour rester compétitives en attendant des réformes plus substantielles de la régulation sociale. Or le moteur de la consommation ne pourra démarrer qu'avec une embellie sur le marché du travail (attendue d'ici la fin de l'année), c'est-à-dire lorsque la reprise des activités se sera traduite en termes d'emploi et que les ménages auront repris confiance en l'avenir.

Le rapport de printemps de la Commission européenne, présenté le 7 avril, apporte une note d'optimisme : après trois ans de non-respect des critères de Maastricht (3,5 \% du PIB en 2002, 3,9\% en 2003, puis 3,6\% cette année), le déficit allemand devrait enfin passer en dessous des $3 \%$ en 2005 pour s'établir à 2,8\%. La Commission a révisé ses prévisions (3,4\% pour 2005 auparavant), convaincue par les efforts de consolidation engagés par le gouvernement Schröder. Mais l'Allemagne n'y parviendra que si les prévisions de croissance (1,5\% cette année et 1,8 \% l'an prochain selon la Commission) se vérifient et à condition que les réformes engagées se traduisent bel et bien par les économies budgétaires escomptées.

En ce printemps 2004, on observe ainsi outre-Rhin deux tendances contradictoires : les fondamentaux reflètent un regain de dynamisme conjoncturel indéniable sur fond de croissance mondiale ; en même temps, les acteurs semblent douter de sa réalité, tant le retour de la croissance et son installation durable dépendent de l'efficience des réformes structurelles en cours et des incertitudes liées à la mutation de l'espace européen. Les 13 échéances électorales de 2004, dont les élections parlementaires européennes en juin, ne sont pas de nature à calmer les crispations. Ce sont elles qui ont mené au récent débat sur les délocalisations industrielles dont le teneur était inhabituelle pour l'Allemagne, le chancelier critiquant publiquement l'absence de « patriotisme social » du patronat. Celui-ci, en la personne de Ludwig Georg Braun, président de la fédération des chambres de commerce et d'industrie DIHK, avait plaidé pour une meilleure prise en compte de la globalisation des
Montée en régime de l'export

Emploi et consommation toujours à la traîne

Retour à la discipline budgétaire européenne

Crispations électoralistes sur les délocalisations 
Elargissement : un risque pour les nouveaux Länder..

... mais une perspective de dynamisation des échanges

Internationalisation : pivot de l'activité outre-Rhin

Elargissement : un révélateur des faiblesses structurelles activités et exhorté les entreprises à « saisir l'opportunité de l'élargissement ». Discours parallèles : le chancelier s'adressait à l'électorat de gauche et à la base syndicale; le patronat pointait du doigt le niveau des prélèvements sociaux et fiscaux pour soutenir l'ardeur réformiste du gouvernement, tout en cherchant à offrir une lecture positive de l'élargissement de l'UE.

Car l'arrivée des 10 nouveaux Etats membres le $1^{\mathrm{er}}$ mai représente une chance pour l'économie allemande. Certes, la concurrence des salaires et des régimes fiscaux se soldera par des difficultés (en Bavière orientale notamment), mais elles devraient rester locales. Plus important est le risque pour les nouveaux Länder qui devront affronter une baisse des fonds structurels européens (voir REA 61/03), alors même que leur dynamisme économique reste faible malgré 1250 milliards $€$ transférés des anciens aux nouveaux Länder dans le cadre de l'effort de reconstruction (1991-2003). C'est d'ailleurs cela qui les pousse aujourd'hui à revendiquer l'adoption d'un nouveau programme de soutien.

Si on fait abstraction de la situation particulière des nouveaux Länder dont certains, soit dit en passant, sont mieux lotis qu'un certain nombre de régions en restructuration à l'Ouest (la Ruhr, par exemple), l'élargissement devrait doper les échanges entre la RFA et les nouveaux Etats membres. Import et export pèsent respectivement quelque $10 \%$ du commerce allemand, soit autant que les échanges avec les USA (voir REA 63/03). Globalement, la croissance des échanges entre anciens et nouveaux membres de l'UE devrait être de $6 \%$ par an dans les dix ans à venir, estime I'Institut IWH. Et, selon l'Agence du commerce extérieur BFAI, les branches motrices de l'économie devraient enregistrer une nette hausse de leurs exportations et de leurs importations à la fois. Ce sont: l'électrotechnique et l'électronique, la chimie, les TIC, la construction mécanique et l'automobile. Quant aux investissements des anciens dans les nouveaux Etats membres de l'UE, ils devraient croître de 5,75\% et 7,25\% en 2004 et 2005 selon la Commission européenne.

A elle seule, la construction mécanique, une branche où dominent les PME, pourrait développer considérablement ses investissements à l'Est - plus pour étendre ses capacités afin de répondre à la forte demande en installations et équipements industriels des nouveaux Etats membres que pour délocaliser des sites de production. Le différentiel salarial et fiscal compte moins dans ce cas que les perspectives de développement des activités. Car en croissant ainsi sur de nouveaux marchés, les entreprises dégagent les marges nécessaires pour investir dans l'innovation et créer des emplois hautement qualifiés sur le sol allemand. C'est de la sorte que, grâce à l'internationalisation de leurs activités et leur positionnement dans une compétition globalisée, les entreprises maintiennent de l'activité outre-Rhin.

Pour l'Allemagne, l'élargissement est aussi un révélateur de ses faiblesses structurelles : celles auxquelles le gouvernement fédéral a commencé à s'attaquer dans le cadre de l'Agenda 2010 (retraites, santé, chômage, fiscalité) et celles auxquelles le chancelier a décidé de remédier dans son discours du 25 mars dernier devant le Bundestag. Si le bilan globalement positif qu'il a livré de l'action gouvernementale s'est attiré les critiques de l'opposition politique, des syndicats, ou des milieux d'affaires, c'est parce que les uns lui reprochent la timidité des réformes, les autres, le « démantèlement des acquis sociaux ». Mais en montrant sa détermination à poursuivre les réformes structurelles et la consolidation budgétaire, le chancelier a rassuré les acteurs économiques. Et il est allé plus loin, en annonçant une politique dédiée à l'innovation.

MAIS L'INNOVATION NE SE RÉSUME PAS À L'INTENTION, réaffirmée, d'investir $3 \%$ du PIB dans la R\&D à l'horizon 2010, ni au développement des politiques sectorielles dans les domaines des hautes technologies, où l'Allemagne est en pointe en Europe. La notion va beaucoup plus loin : elle inclut la modernisation du système scolaire et universitaire dans l'objectif d'une hausse générale des qualifications. C'est l'objectif de Lisbonne, bien sûr, qui est de rendre compétitive l'Europe dans l'économie du savoir. Mais c'est aussi un objectif stratégique pour l'Allemagne si elle veut préserver son avantage compétitif face à dix nouveaux Etats membres au capital humain élevé. C'est encore plus une modernisation sociétale qu'implique l'innovation: la mutation vers une « société apprenante ».

Isabelle Bourgeois (09-04-2004) 